\title{
Impact of clinical features of epidermal growth factor receptor (EGFR)-mutated non-small cell lung cancer (NSCLC) patients on osimertinib efficacy
}

\author{
Chiara Lazzari ${ }^{1}$, Vanesa Gregorc ${ }^{1}$, Mariacarmela Santarpia ${ }^{2}$ \\ ${ }^{1}$ Division of Experimental Medicine, IRCCS San Raffaele Scientific Institute, Milano, Italy; ${ }^{2}$ Medical Oncology Unit, Department of Human \\ Pathology "G. Barresi”, University of Messina, Messina, Italy \\ Correspondence to: Mariacarmela Santarpia, MD, PhD. Medical Oncology Unit, Department of Human Pathology "G. Barresi”, University of \\ Messina, Messina, Italy. Email: msantarpia@unime.it. \\ Provenance: This is an invited article commissioned by the Editorial Office, Fournal of Thoracic Disease. \\ Comment on: Kato Y, Hosomi Y, Watanabe K, et al. Impact of clinical features on the efficacy of osimertinib therapy in patients with T790M-positive \\ non-small cell lung cancer and acquired resistance to epidermal growth factor receptor tyrosine kinase inhibitors. J Thorac Dis 2019;11:2350-60.
}

Submitted Oct 03, 2019. Accepted for publication Oct 16, 2019.

doi: $10.21037 /$ jtd.2019.10.67

View this article at: http://dx.doi.org/10.21037/jtd.2019.10.67

\section{Introduction}

Lung cancer remains the first cause of cancer-related deaths worldwide (1). Thanks to technological advances, the molecular landscape of non-small cell lung cancer (NSCLC) has evolved, with the emergence of different subtypes of NSCLC tumors (2), some of which amenable of targeted inhibition. Clinical practice has shifted towards the era of precision medicine, where molecular classification has become part of routine cancer care, enabling treatment to be matched with patient's tumor genomic profile. In many tumor types, including lung cancer, these efforts have been translated into significantly longer patients' survival (3).

Osimertinib is a third generation epidermal growth factor receptor (EGFR) tyrosine kinase inhibitor (TKI), designed to irreversibly bind the cysteine-797 located in the adenosine triphosphate (ATP)-binding site within the TK domain of the EGFR, thereby determining the inhibition of both EGFR sensitizing- and T790M resistant-mutations (4,5). Moreover, thanks to its physicochemical properties, osimertinib penetrates the blood brain barrier, thus achieving an effective exposure in the central nervous system (CNS) (6). The pronounced clinical activity demonstrated in early phase I and II AURA trials, granted the accelerated approval of osimertinib by the Food and Drug Administration (FDA) in 2015 for the treatment of EGFR T790M mutation-positive NSCLC patients in progression after EGFR TKI therapy (7). In the pooled analysis of phase I and II AURA (8) and AURA2 (9) trials (Table 1), osimertinib was associated with a median overall survival (OS) of 26.8 (10). These positive findings were further confirmed by the phase III AURA3 study (Table 1), that demonstrated the superiority of osimertinib over platinum-pemetrexed chemotherapy in terms of progression free survival (PFS) and objective response rate (ORR), in T790M-positive advanced NSCLC patients who had progressed to first-line EGFR-TKIs, including in the subgroup of patients with brain metastases $(12,13)$. Currently, EGFR T790M mutation status can be performed by using either a validated plasma-based or a tissue-based test. However, if the result of plasma detection in plasma is negative, due to the potential for false negative results, a tissue test is advisable wherever possible to determine EGFR T790M mutation (7).

\section{Osimertinib in a real-world population of EGFR- mutant NSCLC patients}

In order to define the efficacy of osimertinib in a realworld population and identify the prognostic impact of clinical features on patients' outcome, Kato and colleagues retrospectively analyzed 31 EGFR-mutated patients, developing the T790M resistance mutation following progression to first- and second-generation EGFR-TKIs, who received osimertinib between March 2016 and January 2018 (11). Among the patients enrolled, 74.2\% were elderly 
Table 1 results from phase 1, phase 2 and phase 3 studies investigating the efficacy of osimertinib

\begin{tabular}{|c|c|c|c|c|}
\hline Study (ref) & Treatment & Number of patients & ORR (\%), OR (95\% Cl) & PFS (months), HR (95\% Cl) \\
\hline AURA2 (phase 2) (8) & Osimertinib & 210 T790M+ & $70(64-77)$ & $9.9(8.5-12.3)$ \\
\hline AURA 3 (phase 3) (10) & $\begin{array}{l}\text { Osimertinib vs. } \\
\text { platinum-pemetrexed }\end{array}$ & 419 T790M+ & $\begin{array}{l}71 \text { vs. } 31 \\
\text { OR 5.39, (3.47-8.48) } P<0.001\end{array}$ & $\begin{array}{l}10.1 \text { vs. } 4.4 \\
\text { HR } 0.30,(0.23-0.41) \mathrm{P}<0.001\end{array}$ \\
\hline
\end{tabular}

ORR, objective response rate; OR, odds ratio; PFS, progression free survival; HR, hazard ratio.

( $\geq 65$ years), $32.3 \%$ had a poor ECOG-performance staus (PS) of $2-4$, and $45.2 \%$ brain metastases. In most of these cases $(67.7 \%)$, T790M mutation was detected with liquid biopsy. After a median duration of follow up of 12 months, the median PFS observed was 5.6 months and the median OS was 19.4 months. PFS and OS were longer in elderly compared with young patients, and in those with a good ECOG-PS. At the univariate analysis, age and ECOG-PS were the only clinical features associated with prognosis, that remained independent predictors of osimertinib efficacy at the multivariate analysis.

\section{Comments and future perspectives}

Conversely from the results of the AURA3 study, where a median PFS of 10.1 months was registered in patients receiving osimertinib, the median PFS of patients included in this retrospective analysis was significantly shorter (5.6 months). These findings might be partly explained by the different populations enrolled: median age of patients included in the AURA3 was 62 years, while the median age of those included by Kato and colleagues was 72 years. Moreover, more patients had a poor ECOG PS compared to the patients enrolled in the AURA3, where only $6.8 \%$ had an ECOG PS of 2. Finally, 71\% of the patients evaluated by Kato and colleagues had received platinumbased doublet chemotherapy before starting treatment with osimertinib, thus being a more pretreated population.

At the univariate and multivariate analysis, ECOG PS and age were the only two clinical features significantly associated with patients' outcome. The PS reflects both tumor burden and patients' co-morbidities and represents the assessment of the patients' level of function and capability of self-care (14). It is an important clinical tool for measuring quality of life among cancer patients and, so far, has been recognized as one of the most important prognostic factors in cancer patients, influencing clinicians' treatment decisions. Patients with poor PS generally exhibit a dismal prognosis independent of treatment. However, it can be speculated that an intricate network mediated by angiogenesis and the immune system might enhance cancer progression and confer resistance to osimertinib in EGFR-mutated NSCLC patients with poor PS.

A serum proteomic test, VeriStrat ${ }^{\circledR}$, based on $8 \mathrm{~m} / \mathrm{z}$ spectrometry peaks, able to classify NSCLC patients into two groups (VeriStrat ${ }^{\circledR}$ good or VeriStrat ${ }^{\circledR}$ poor), was initially built to identify those NSCLC patients who could have benefit from first generation EGFR-TKIs (15). At that time, the predictive value of EGFR activating mutations was still not clear. In the PROSE study, including patients who received chemotherapy or erlotinib, the VeriStrat ${ }^{\circledR}$ demonstrated to be a potential prognostic and predictive tool to be used in clinical setting. Indeed, a significant longer OS was observed in VeriStrat ${ }^{\circledR}$ good classified patients, compared with the poor (11.0 months and 3.7 months, respectively), therefore suggesting the clinical value of VeriStrat ${ }^{\circledR}$ to identify those patients with short life expectancy and unsuitable to receive further treatments (16). Moreover, its predictive value was also demonstrated, as patients with a proteomic test classification of poor had worse survival on erlotinib than on chemotherapy, while no significant difference in OS was observed between treatments for those with a proteomic test classification of good. When the mass spectrometry peaks were investigated at the molecular level, four out of the eight $\mathrm{m} / \mathrm{z}$ peaks distinguishing VeriStrat ${ }^{\circledR}$ poor and good proteomic profiles were generated by serum amyloid a protein 1 (SAA1) and its two truncated forms (17). SAA1 is an acute phase protein, induced by interleukin-1 (IL-1), interleukin-6 (IL-6) and tumor necrosis factor alpha $(\mathrm{TNF}-\alpha)(18)$. Data from literature indicate that IL-6 reduces the dependence of survival signals from the EGFR pathway through an increased autocrine stimulation of the IL-6/gp130/ signal transducer and activator of transcription 3 (STAT3) signaling $(19,20)$. IL-6 activates the Janus kinase (JAK) and Src kinase, that are responsible for STAT3 phosphorylation. Once phosphorylated, STAT3 translocates to the nucleus, 
and activates the transcription of genes involved in cell cycle progression (cyclin D1, survivin), cell survival (B cell leukemia/ lymphoma 2), angiogenesis, including the vascular endothelial growth factor A (VEGFA) and immune suppression, as the programmed death ligand-1 (PD-L1). In EGFR-mutated NSCLC patients with poor ECOG PS, multiple genes involved in angiogenesis and immune response may play a role in cancer progression and affect osimertinib efficacy.

With regards to the impact of age on patients' prognosis, data from literature indicate controversial results. The poor prognosis observed in the younger patients included in the analysis by Kato and colleagues might be the result of a different genomic complexity distinguishing younger from elderly. It is currently known that EGFR-mutated NSCLC is as a heterogeneous disease, including subsets of molecularly subcategorized patients. Data from literature indicate that young patients harbor more frequently TP53 mutations compared with elderly (21). It is possible that some heterogeneous preexisting subclones in younger patients might emerge under pressure of TKI therapy and confer resistance to treatment, thus likely explaining the poor prognosis. Recently, concurrent mutations in TP53, phosphatidylinositol-4,5-bisphosphate 3-kinase catalytic subunit alpha (PIK3CA), catenin beta-1 (CTNNB1) and retinoblastoma (RB1) were observed in EGFR-mutated NSCLC. The presence of TP53 mutations was associated with shorter OS and PFS. Loss of function mutations in TP53 and RB1, identified in approximately nine percent of EGFR-mutated patients, increases the risk of small cell lung cancer (SCLC) transformation. Similarly, concomitant amplification in mesenchymal-epithelial transition factor (MET) or ERBB2 genes significantly reduces PFS in patients under EGFR-TKIs, thus suggesting the need to define customized combinatorial treatment strategies (22).

Following the results of the FLAURA study, showing significant prolonged PFS and better safety profile with osimertinib over standard EGFR-TKI, and a significantly lower percentage of CNS progression in the osimertinib arm (23), osimertinib indication was extended as firstline treatment of EGFR-mutated NSCLC patients, thus improving the therapeutic opportunities and opening new questions regarding its use up-front or at the time of disease progression following treatment with first- or second-generation EGFR-TKIs. The PFS improvement of osimertinib over first generation-TKIs observed in the FLAURA and the early separation of the Kaplan-Meyer curves, documented at the first computed tomography scan evaluation, might be the result of the efficient inhibition of osimertinib on pretreatment EGFR T790M. It is documented that a subpopulation of patients can harbor EGFR T790M at baseline. Pretreatment T790M was not evaluated in patients enrolled in the FLAURA study. Available data from liquid biopsy performed in patients developing progression under osimertinib, indicate that up-front osimertinib prevents T790M-mediated resistance, as no evidence of T790M was identified, thus confirming the efficient inhibition of T790M clones. Multiple biological mechanisms of acquired resistance to osimertinib have been identified, including secondary resistance mutations, that interfere with drug binding, or the activation of alternative signaling pathways $(24,25)$. The most common resistance mechanisms resulted to be the C797S mutation (7\%) and MET amplification (15\%). Other mechanisms included HER2 amplification, PIK3CA, KRAS, BRAF or rare EGFR secondary mutations. Based on these findings, different phase I/II trials are exploring the safety of combinatorial approaches, including osimertinib and targeted agents directed against the activated bypass signaling pathway, that may potentially improve patients' outcome. Molecular profiling of tumors on tissue or liquid biopsy may provide genetic signatures underlying intrinsic and acquired resistance to osimertinib and suggest novel potential effective therapeutic combinations.

\section{Acknowledgments}

None.

\section{Footnotes}

Conflicts of Interest: The authors have no conflicts of interest to declare.

Ethical Statement: The author is accountable for all aspects of the work in ensuring that questions related to the accuracy or integrity of any part of the work are appropriately investigated and resolved

\section{References}

1. Torre LA, Siegel RL, Ward EM, et al. Global Cancer Incidence and Mortality Rates and Trends--An Update. Cancer Epidemiol Biomarkers Prev 2016;25:16-27.

2. Rosell R, Karachaliou N. Large-scale screening for somatic mutations in lung cancer. Lancet 2016;387:1354-6.

3. Kris MG, Johnson BE, Berry LD, et al. Using multiplexed assays of oncogenic drivers in lung cancers to select 
targeted drugs. JAMA 2014;311:1998-2006.

4. Cross DA, Ashton SE, Ghiorghiu S, et al. AZD9291, an irreversible EGFR TKI, overcomes T790M-mediated resistance to EGFR inhibitors in lung cancer. Cancer Discov 2014;4:1046-61.

5. Finlay MR, Anderton M, Ashton S, et al. Discovery of a potent and selective EGFR inhibitor (AZD9291) of both sensitizing and T790M resistance mutations that spares the wild type form of the receptor. J Med Chem 2014;57:8249-67.

6. Zeng Q, Wang J, Cheng Z, et al. Discovery and Evaluation of Clinical Candidate AZD3759, a Potent, Oral Active, Central Nervous System-Penetrant, Epidermal Growth Factor Receptor Tyrosine Kinase Inhibitor. J Med Chem 2015;58:8200-15.

7. Santarpia M, Liguori A, Karachaliou N, et al. Osimertinib in the treatment of non-small-cell lung cancer: design, development and place in therapy. Lung Cancer (Auckl) 2017;8:109-125.

8. Janne PA, Yang JC, Kim DW, et al. AZD9291 in EGFR inhibitor-resistant non-small-cell lung cancer. N Engl J Med 2015;372:1689-99.

9. Goss G, Tsai CM, Shepherd FA, et al. Osimertinib for pretreated EGFR Thr790Met-positive advanced nonsmall-cell lung cancer (AURA2): a multicentre, open-label, single-arm, phase 2 study. Lancet Oncol 2016;17:1643-52.

10. Ahn MJ, Tsai CM, Shepherd FA, et al. Osimertinib in patients with T790M mutation-positive, advanced nonsmall cell lung cancer: Long-term follow-up from a pooled analysis of 2 phase 2 studies. Cancer 2019;125:892-901.

11. Kato Y, Hosomi Y, Watanabe K, et al. Impact of clinical features on the efficacy of osimertinib therapy in patients with T790M-positive non-small cell lung cancer and acquired resistance to epidermal growth factor receptor tyrosine kinase inhibitors. J Thorac Dis 2019;11:2350-60.

12. Wu YL, Ahn MJ, Garassino MC, et al. CNS Efficacy of Osimertinib in Patients With T790M-Positive Advanced Non-Small-Cell Lung Cancer: Data From a Randomized Phase III Trial (AURA3). J Clin Oncol 2018;36:2702-9.

13. Mok TS, Wu YL, Ahn MJ, et al. Osimertinib or PlatinumPemetrexed in EGFR T790M-Positive Lung Cancer. N Engl J Med 2017;376:629-40.

14. Sorensen JB, Klee M, Palshof T, et al. Performance status assessment in cancer patients. An inter-observer variability study. Br J Cancer 1993;67:773-5.

15. Taguchi F, Solomon B, Gregorc V, et al. Mass spectrometry to classify non-small-cell lung cancer patients for clinical outcome after treatment with epidermal growth factor receptor tyrosine kinase inhibitors: a multicohort cross- institutional study. J Natl Cancer Inst 2007;99:838-46.

16. Gregorc V, Novello S, Lazzari C, et al. Predictive value of a proteomic signature in patients with non-small-cell lung cancer treated with second-line erlotinib or chemotherapy (PROSE): a biomarker-stratified, randomised phase 3 trial. Lancet Oncol 2014;15:713-21.

17. Milan E, Lazzari C, Anand S, et al. SAA1 is over-expressed in plasma of non small cell lung cancer patients with poor outcome after treatment with epidermal growth factor receptor tyrosine-kinase inhibitors. J Proteomics 2012;76:91-101.

18. Weinstein PS, Skinner M, Sipe JD, et al. Acute-phase proteins or tumour markers: the role of SAA, SAP, CRP and CEA as indicators of metastasis in a broad spectrum of neoplastic diseases. Scand J Immunol 1984;19:193-8.

19. Chaib I, Karachaliou N, Pilotto S, et al. Co-activation of STAT3 and YES-Associated Protein 1 (YAP1) Pathway in EGFR-Mutant NSCLC. J Natl Cancer Inst 2017;109. doi: 10.1093/jnci/djx014.

20. Codony-Servat C, Codony-Servat J, Karachaliou N, et al. Activation of signal transducer and activator of transcription 3 (STAT3) signaling in EGFR mutant non-small-cell lung cancer (NSCLC). Oncotarget 2017;8:47305-16.

21. Giordano M, Boldrini L, Servadio A, et al. Differential microRNA expression profiles between young and old lung adenocarcinoma patients. Am J Transl Res 2018;10:892-900.

22. Yu HA, Suzawa K, Jordan E, et al. Concurrent Alterations in EGFR-Mutant Lung Cancers Associated with Resistance to EGFR Kinase Inhibitors and Characterization of MTOR as a Mediator of Resistance. Clin Cancer Res 2018;24:3108-18.

23. Soria JC, Ohe Y, Vansteenkiste J, et al. Osimertinib in Untreated EGFR-Mutated Advanced Non-Small-Cell Lung Cancer. N Engl J Med 2018;378:113-25.

24. Ortiz-Cuaran S, Scheffler M, Plenker D, et al. Heterogeneous Mechanisms of Primary and Acquired Resistance to Third-Generation EGFR Inhibitors. Clin Cancer Res 2016;22:4837-47.

25. Lazzari C, Gregorc V, Karachaliou N, et al. Mechanisms of resistance to osimertinib. J Thorac Dis 2019. doi: 10.21037/jtd.2019.08.30

Cite this article as: Lazzari C, Gregorc V, Santarpia $M$. Impact of clinical features of epidermal growth factor receptor (EGFR)-mutated non-small cell lung cancer (NSCLC) patients on osimertinib efficacy. J Thorac Dis 2019;11(11):4400-4403. doi: $10.21037 /$ jtd.2019.10.67 